International Journal of Applied Mathematical Research, 6(4) (2017) 125-129
International Journal of Applied Mathematical Research
SPC
Website: www.sciencepubco.com/index.php/IJAMR
doi: $10.14419 /$ ijamr.v6i4.8198
Research paper

\title{
Bivariate Legendre approximation
}

\author{
Ismahene Sehili $^{1 *}$ and Abdelhamid Zerroug ${ }^{2}$ \\ ${ }^{1}$ Department of Mathematics, University of Biskra, Algeria \\ ${ }^{2}$ Department of Mathematics, University of Biskra, Algeria \\ ${ }^{*}$ Corresponding authorE-mail: sehilimath@gmail.com
}

\begin{abstract}
Spectral methods are among the numerical methods commonly used for approximating solutions of boundary value problems. In this paper we propose, a generalization of the spectral Tau method in dimension 2, this method is generalized by the use of a new two-dimensional polynomial basis constructed by a three terms recurrence relation. We also present an estimation of error committed by the proposed method.
\end{abstract}

Keywords: Two-dimensional Legendre basis, Three terms recurrence construction, Error Estimation.

\section{Introduction}

Primarily in computational applications, functions of two variables often need to be approximated by other functions that are better understood or more readily evaluated. Polynomials are among the simplest mathematical functions which have the flexibility to represent very general nonlinear relationships. Approximation of more complicated functions by polynomials is a basic building block for many numerical techniques [7].

Two-dimensional approximation is an extension of one-dimensional approximation for approximating functions of two variables. Generally, the key idea is to perform one-dimensional approximation first in one variable, and then again in the other variable. Although each step is one-dimensional.

In [1], the authors expressed the solution function with the twodimensional Legendre polynomials produced by a tensor-product. The approximate solution is

$U_{N}(x, y)=\sum_{i=1}^{N} \sum_{j=1}^{N} \hat{U}_{i, j} L_{i}(x) L_{j}(y) \quad i, j=0, \ldots, N$.

where $L_{i}$ is the Legendre polynomial of degree $i$.

But with the advantage of multi-dimensional polynomial basis, the solution can be expanded to a multi-dimensional basis function to have good approximations for solutions of multi-dimensional problems.

As a first part, we have developed a new orthogonal polynomial basis by a three term recurrence relation. We have also demonstrate the orthogonality of these polynomials using a property defined inner product on the space of two-dimensional polynomials

$\left\langle P_{i, j}, P_{l, k}\right\rangle=\int_{-1}^{1} \int_{-1}^{1} P_{i, j}(x, y) P_{l, k}(x, y) d x d y$.

Numerical stability is a global property of the numerical algorithms, a necessary quality to hope getting results have meaning. In the second part of this work, we present a theoretical result concerning the stability of the spectral method discussed.

\section{Construction and properties}

\subsection{Recursive construction}

The idea of modeling by orthogonal polynomials is to approximate real functions by linear combinations of two-dimensional polynomial functions, for example those of Legendre. We use the bivariate polynomials defined in $\mathbb{R}^{2}$ as follows :

$P_{K, L}(x, y)=\sum_{k=0}^{K} \sum_{l=0}^{L} a_{k, l}(x)^{k}(y)^{l}$,

with $K \in \mathbb{N}^{+}$is the maximum degree of $x, L \in \mathbb{N}^{+}$the maximum degree of $y$ and $c_{k, l}$ the all real coefficients of the polynomial. The degree of the polynomial is $K+L$. We define an inner product by

$\left\langle F_{1}(x, y), F_{2}(x, y)\right\rangle=\iint_{\Omega} F_{1}(x, y) F_{2}(x, y) \omega(x, y) d x d y$,

with $\Omega$ is the domain of definition and $\omega(x, y)$ is the weight function of the inner product. We can build a two-dimensional Legendre basis by the following three terms recurrence formula

$$
\left\{\begin{array}{l}
P_{-1, j}(x, y)=0 \\
P_{i,-1}(x, y)=0 \\
P_{0,0}(x, y)=1 \\
P_{i+1, j}(x, y)=\frac{2 i+1}{i+1} x P_{i, j}(x, y)-\frac{i}{i+1} P_{i-1, j}(x, y) \\
P_{i, j+1}(x, y)=\frac{2 j+1}{j+1} y P_{i, j}(x, y)-\frac{j}{j+1} P_{i, j-1}(x, y)
\end{array}\right.
$$

It is important to note that the weight function of the inner product $\omega(x, y)$ is equal to 1 , this makes the computing of the inner product easier and faster, unlikely for many bases of orthogonal polynomials that have weight functions which requires considerable time for the computation. The inner product in the Legendre polynomials basis can be calculated by evaluating only two functions instead of three. 


\subsection{Basic formulas.}

We present here a collection of the essential formulas for twodimensional Legendre polynomials.

- Two-dimensional Legendre Polynomials may be expressed using this generalisation of Rodrigues formula

$$
P_{i, j}(x, y)=\frac{1}{2^{i+j} i ! j !} \frac{\partial^{i}}{\partial x^{i}}\left(x^{2}-1\right)^{i} \frac{\partial^{j}}{\partial y^{j}}\left(y^{2}-1\right)^{j}
$$

- $P_{i, j}(1,1)=1, \quad \forall i, j \geq 0$

- $P_{i, j}(-1,-1)=(-1)^{i+j}, \quad \forall i, j>0$.

- $\left\|P_{i, j}(x, y)\right\| \leq 1, \quad(-1,-1) \leq(x, y) \leq(1,1)$.

- A two-dimensional basis of degree $d$ can be composed by the Legendre polynomials $\left\{P_{i, j}\right\}$ with $i+j \leq d$.

- The number of polynomials that make up the basis of degree $d$ is $n_{d}=\frac{(d+1)(d+2)}{2}$

- The domain orthogonality of the two-dimensional Legendre polynomials is $\Omega \in[-1,1]^{2}$.

- $\int_{-1}^{1} \int_{-1}^{1} P_{i, j}^{2} d x d y=\left(i+\frac{1}{2}\right)^{-1}\left(j+\frac{1}{2}\right)^{-1}$;

The key to proving this last formula is

$$
\left\{\begin{aligned}
i P_{i, j}(x, y) & =x \frac{\partial P_{i, j}}{\partial x}(x, y)-\frac{\partial P_{i-1, j}}{\partial x}(x, y) \\
j P_{i, j}(x, y) & =y \frac{\partial P_{i, j}}{\partial x}(x, y)-\frac{\partial P_{i, j-1}}{\partial x}(x, y) .
\end{aligned}\right.
$$

- We present an establishment of the two-dimensional Legendre polynomials (Matlab source code)

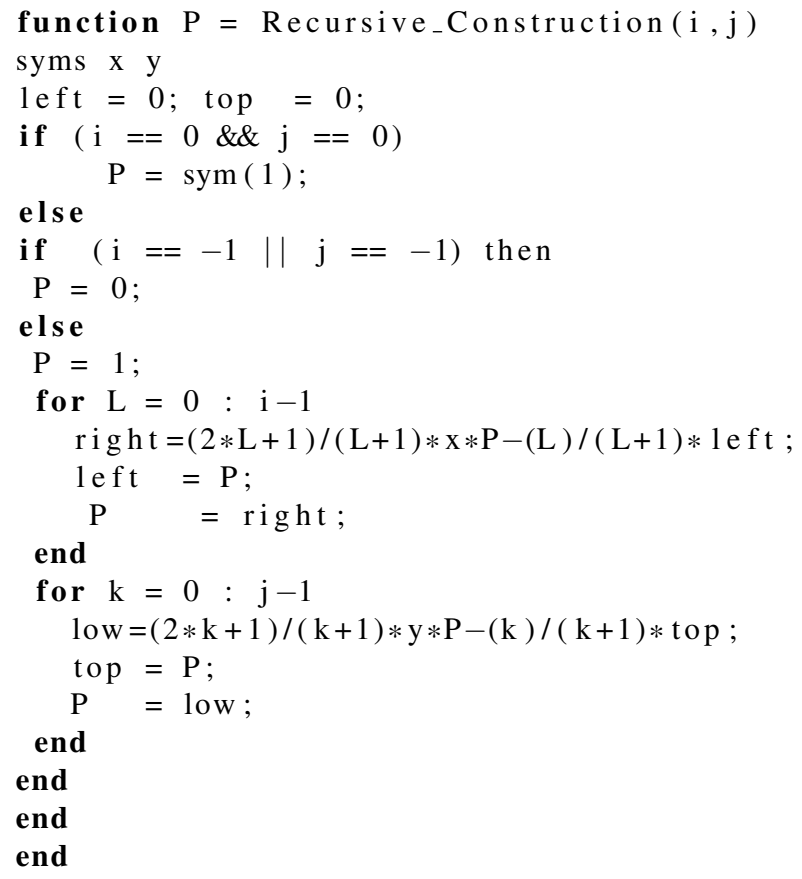

The first polynomial $P_{0,0}$ is located in the upper left corner. An evolution along the lines of the same column does not vary the degree of the polynomials according to $x$. An evolution depending on the column of the same line varies depending on the degree $y$

- Differentiation is to calculate the Legendre development of the derivative of a function expressed as a combination of the two-dimensional Legendre polynomials [4]. If

$$
U_{N}(X, t)=\sum_{i=0}^{N} \sum_{j=0}^{N-i} \hat{U}_{i, j}(t) P_{i, j}(x, y), \quad X \in \mathbb{R}^{n} \times \mathbb{R}^{n},
$$

by the recurrence relation

$$
\left\{\begin{aligned}
(2 i+1) P_{i, j}(x, y) & =\frac{\partial}{\partial x} P_{i+1, j}(x, y)-\frac{\partial}{\partial x} P_{i-1, j}(x, y) \\
(2 j+1) P_{i, j}(x, y) & =\frac{\partial}{\partial y} P_{i, j+1}(x, y)-\frac{\partial}{\partial y} P_{i, j-1}(x, y),
\end{aligned}\right.
$$

we can prove that the coefficients of the first derivative are given by

$$
\hat{U}_{i, j}^{(1)}=\left\{\begin{array}{l}
(2 i+1)\left(\sum_{\substack{p=i+1 \\
p+i \text { odd }}} \hat{U}_{i, j}\right), \quad i, j \geq 0, \\
(2 j+1)\left(\sum_{\substack{p=j+1 \\
p+j \text { odd }}} \hat{U}_{i, j}\right), \quad i, j \geq 0,
\end{array}\right.
$$

The previous identity generalizes, with obvious notation to

$$
\hat{U}_{i, j}^{(q-1)}= \begin{cases}\frac{\hat{U}_{i-1, j}^{(q)}}{2 i-1}-\frac{\hat{U}_{i+1, j}^{(q)}}{2 i+3} \quad i \geq 1, \\ \frac{\hat{U}_{i, j-1}^{(q)}-\frac{\hat{U}_{i, j+1}^{(q)}}{2 j-1}}{2 j+3} \quad j \geq 1,\end{cases}
$$

from which it is possible to get explicit expressions for the Legendre coefficient of higher derivatives.

For the second derivative we have

$$
\hat{U}_{i, j}^{(2)}=\left\{\begin{array}{l}
\left(i+\frac{1}{2}\right)\left(\sum_{\substack{p=i+2 \\
p+i \text { even }}}[p(p+1)-i(i+1)] \hat{U}_{i, j}\right), \quad i, j \geq 0, \\
\left(j+\frac{1}{2}\right)\left(\sum_{\substack{p=j+2 \\
p+j \text { even }}}[p(p+1)-j(j+1)] \hat{U}_{i, j}\right), \quad i, j \geq 0,
\end{array}\right.
$$

The previous expansions are not merely formal provided $U$ is smooth enough.

\subsection{Orthogonality}

Two-dimensional Legendre polynomials satisfy the following differential equation

$$
\begin{aligned}
& \frac{\partial}{\partial x}\left[\left(1-x^{2}\right) \frac{\partial P_{i, j}}{\partial x}(x, y)\right]+\frac{\partial}{\partial y}\left[\left(1-y^{2}\right) \frac{\partial P_{i, j}}{\partial y}(x, y)\right] \\
&+\quad[i(i+1)+j(j+1)] P_{i, j}(x, y)=0
\end{aligned}
$$

In this section, we prove the orthogonality of two-dimensional Legendre polynomials, $\mathrm{i}$ e, we prove that

$\left\langle P_{i, j}, P_{l, k}\right\rangle=0 \quad$ for $\quad(i, j) \neq(l, k)$.

Proof. Using the inner product (2)

Let $P_{i, j}$ and $P_{l, k}$ be a two-dimensional Legendre polynomials of degree $i+j$ and $l+k$ respectively, then, they satisfy the equation (4)

$$
\begin{aligned}
& \frac{\partial}{\partial x}\left[\left(1-x^{2}\right) \frac{\partial P_{i, j}}{\partial x}(x, y)\right]+\frac{\partial}{\partial y}\left[\left(1-y^{2}\right) \frac{\partial P_{i, j}}{\partial x}(x, y)\right] \\
= & -[i(i+1)+j(j+1)] P_{i, j}(x, y) \\
& \frac{\partial}{\partial x}\left[\left(1-x^{2}\right) \frac{\partial P_{l, k}}{\partial x}(x, y)\right]+\frac{\partial}{\partial y}\left[\left(1-y^{2}\right) \frac{\partial P_{l, k}}{\partial x}(x, y)\right] \\
= & -[l(l+1)+k(k+1)] P_{l, k}(x, y)
\end{aligned}
$$


Multiplying (5) by $P_{l, k}$ and (6) by $P_{i, j}$ and subtracting

$$
\begin{aligned}
& {[(l(l+1)+k(k+1))-(i(i+1)+j(j+1))] P_{i, j}(x, y) P_{l, k}(x, y)=} \\
& {\left[\frac{\partial}{\partial x}\left[\left(1-x^{2}\right) \frac{\partial P_{i, j}}{\partial x}(x, y)\right]+\frac{\partial}{\partial y}\left[\left(1-y^{2}\right) \frac{\partial P_{i, j}}{\partial y}(x, y)\right]\right] P_{l, k}(x, y)-} \\
& {\left[\frac{\partial}{\partial x}\left[\left(1-x^{2}\right) \frac{\partial P_{l, k}}{\partial x}(x, y)\right]+\frac{\partial}{\partial y}\left[\left(1-y^{2}\right) \frac{\partial P_{l, k}}{\partial y}(x, y)\right]\right] P_{i, j}(x, y)}
\end{aligned}
$$

We set

$c=\left[l^{2}+l-i^{2}-i\right]+\left[k^{2}+k-l^{2}-l\right] \neq 0, \quad$ if $\quad(l, k) \neq(i, j)$.

By integrating of both members of $(7)$ in the domain $[-1,1] \times[-1,1]$

$$
\begin{aligned}
& c \int_{-1}^{1} \int_{-1}^{1} P_{i, j} P_{l, k} d x d y \\
= & \int_{-1}^{1} \int_{-1}^{1}\left[\left[\frac{\partial}{\partial x}\left[\left(1-x^{2}\right) \frac{\partial P_{i, j}}{\partial x}\right]+\frac{\partial}{\partial y}\left[\left(1-y^{2}\right) \frac{\partial P_{i, j}}{\partial y}\right]\right] P_{l, k}\right] \\
- & {\left[\left[\frac{\partial}{\partial x}\left[\left(1-x^{2}\right) \frac{\partial P_{l, k}}{\partial x}\right]+\frac{\partial}{\partial y}\left[\left(1-y^{2}\right) \frac{\partial P_{l, k}}{\partial y}\right]\right] P_{i, j}\right] d x d y }
\end{aligned}
$$

We start by the first part

$$
\begin{aligned}
I_{1}= & \int_{-1}^{1} \int_{-1}^{1}\left[\left[\frac{\partial}{\partial x}\left[\left(1-x^{2}\right) \frac{\partial P_{i, j}}{\partial x}\right]+\frac{\partial}{\partial y}\left[\left(1-y^{2}\right) \frac{\partial P_{i, j}}{\partial y}\right]\right] P_{l, k}\right] d x d y_{1} \\
= & \int_{-1}^{1} \int_{-1}^{1}\left[\frac{\partial}{\partial x}\left[\left(1-x^{2}\right) \frac{\partial P_{i, j}}{\partial x}\right]\right] P_{l, k} d x d y \\
& +\int_{-1}^{1} \int_{-1}^{1}\left[\frac{\partial}{\partial y}\left[\left(1-y^{2}\right) \frac{\partial P_{i, j}}{\partial y}\right]\right] P_{l, k} d x d y
\end{aligned}
$$

We have

$$
\begin{aligned}
I_{1,1}= & \int_{-1}^{1}\left[\int_{-1}^{1}\left[\frac{\partial}{\partial x}(x, y)\left[\left(1-x^{2}\right) \frac{\partial P_{i, j}}{\partial x}(x, y)\right]\right] P_{l, k}(x, y) d x\right] d y \\
= & \int_{-1}^{1}\left[\left[P_{l, k}(x, y)\left(1-x^{2}\right) \frac{\partial P_{i, j}}{\partial x}(x, y)\right]_{-1}^{1}\right. \\
& \left.-\int_{-1}^{1} \frac{\partial P_{l, k}}{\partial x}(x, y)\left(1-x^{2}\right) \frac{\partial P_{i, j}}{\partial x}(x, y) d x\right] d y
\end{aligned}
$$

hence

$$
I_{1,1}=-\int_{-1}^{1}\left[\int_{-1}^{1}\left[\frac{\partial P_{l, k}}{\partial x}(x, y)\left(1-x^{2}\right) \frac{\partial P_{i, j}}{\partial x}(x, y) d x\right]\right] d y
$$

Similarly

$$
\begin{aligned}
I_{1,2} & =\int_{-1}^{1}\left[\int_{-1}^{1}\left[\frac{\partial}{\partial y}\left[\left(1-y^{2}\right) \frac{\partial P_{i, j}}{\partial y}(x, y)\right]\right] P_{l, k}(x, y) d y\right] d x \\
& =-\int_{-1}^{1}\left[\int_{-1}^{1} \frac{\partial P_{l, k}}{\partial x}(x, y)\left(1-y^{2}\right) \frac{\partial P_{i, j}}{\partial x}(x, y) d x\right] d y
\end{aligned}
$$

For the second part

$$
\begin{gathered}
I_{2}=\int_{-1}^{1} \int_{-1}^{1}\left[\frac{\partial}{\partial x}\left[\left(1-x^{2}\right) \frac{\partial P_{l, k}}{\partial x}\right] P_{i, j}\right] d x d y \\
\left.+\int_{-1}^{1} \int_{-1}^{1}\left[\frac{\partial}{\partial y}\left[\left(1-y^{2}\right) \frac{\partial P_{l, k}}{\partial y}\right]\right] P_{i, j}\right] d x d y \\
I_{2,1}=\int_{-1}^{1}\left[\int_{-1}^{1}\left[\frac{\partial}{\partial x}\left[\left(1-x^{2}\right) \frac{\partial P_{l, k}}{\partial x}(x, y)\right]\right] P_{i, j}(x, y) d x\right] d y \\
=-\int_{-1}^{1}\left[\int_{-1}^{1} \frac{\partial P_{i, j}}{\partial x}(x, y)\left(1-x^{2}\right) \frac{\partial P_{l, k}}{\partial x}(x, y) d x\right] d y
\end{gathered}
$$

and also

$$
\begin{aligned}
I_{2,2} & =\int_{-1}^{1}\left[\int_{-1}^{1}\left[\frac{\partial}{\partial y}\left[\left(1-y^{2}\right) \frac{\partial P_{l, k}}{\partial y}(x, y)\right]\right] P_{i, j}(x, y) d y\right] d x \\
& =-\int_{-1}^{1}\left[\int_{-1}^{1} \frac{\partial P_{i, j}}{\partial y}(x, y)\left(1-y^{2}\right) \frac{\partial P_{l, k}}{\partial y}(x, y) d y\right] d x
\end{aligned}
$$

therefore

$$
\begin{aligned}
c \int_{-1}^{1} \int_{-1}^{1} P_{i, j}(x, y) P_{l, k}(x, y) d x d y & =I_{1}-I_{2} \\
& =\left[I_{1,1}+I_{1,2}\right]-\left[I_{2,1}+I_{2,2}\right] \\
& =0 .
\end{aligned}
$$

and since $c \neq 0$ for every pair $(i, j) \neq(l, k)$, then

$$
\begin{aligned}
\int_{-1}^{1} \int_{-1}^{1} P_{i, j}(x, y) P_{l, k}(x, y) d x d y & =0 \\
\left\langle P_{i, j}, P_{l, k}\right\rangle & =0, \quad \text { for }(i, j) \neq(l, k) .
\end{aligned}
$$

hence the result.

\subsection{Numerical tests.}

In order to give a first illustration of the performance of our implementation of bivariate Legendre approximation, we apply the method to some bivarite functions.

$d y$ Table (1) reports the results corresponding to the application of the approximation algorithm at various order (application of the method in $[-1 ; 1]^{2}$ ). The absolute and relative errors have been computed with a suitable norm.

Table 1: Absolute and relative approximation error.

\begin{tabular}{cccc}
\hline $\mathbf{f}(\mathbf{x}, \mathbf{y})$ & $\mathbf{N}$ & Absolute error & Relative error \\
\hline \hline & $2^{0}$ & 0.6387 & 0.4111 \\
& $2^{1}$ & 0.3343 & 0.2152 \\
$\cos (x+y)$ & $2^{2}$ & 0.0359 & 0.0231 \\
& $2^{3}$ & 0 & 0 \\
\hline & $2^{0}$ & 1.8115 & 0.4995 \\
$\exp (x+y)$ & $2^{2}$ & 0.6158 & 0.1698 \\
& $2^{2}$ & 0.1022 & 0.0282 \\
& $2^{3}$ & 0.0014 & $3.8565 \times 10^{-4}$ \\
\hline
\end{tabular}

For the sake of comparison with another widely used bivariate method (least squares method), we show in Table (2) the results of an approximation in another basis (basis constructed by a tensor product of the Chebychev polynomials) [4]-[10]

Table 2: Square approximation error for some functions.

\begin{tabular}{cccc}
\hline $\mathbf{f}(\mathbf{x}, \mathbf{y})$ & $\mathbf{N}$ & Legendre Error & Chebychev Error \\
\hline \multirow{3}{*}{$x^{2} y+y^{2} x$} & $2^{0}$ & 0.4869 & 0.5196 \\
& $2^{1}$ & 0.3443 & 0.4732 \\
& $2^{2}$ & 0 & 0.4218 \\
\hline \multirow{4}{*}{$y \cos (x)$} & $2^{0}$ & 0.9848 & 0.9958 \\
& $2^{1}$ & 0.1602 & 0.3613 \\
& $2^{2}$ & $35 \times 10^{-4}$ & 0.5389 \\
\hline \hline $\sin \pi x \sin \pi y$ & $2^{3}$ & $3.0037 \times 10^{-5}$ & 1.4443 \\
\hline & $2^{0}$ & 1 & 1 \\
& $2^{1}$ & 0.7940 & 0.8628 \\
& $2^{2}$ & 0.6304 & 0.6439 \\
& $2^{3}$ & 0.1322 & 0.6853 \\
& $2^{4}$ & $3 \times 10^{-4}$ & 0.8144 \\
\hline
\end{tabular}

Practical implementation of the bivariate Legendre approximation method follows strictly the construction in (3). 


\section{Tau-Legendre approximation}

\subsection{Theoretical background}

Let the universe of our discussion be the Hilbert space $\mathscr{H}:=$ $\mathscr{L}^{2}([-1,1] \times[-1,1], \mathbb{C})$. We wish to obtain an easily implantable computer model and to process large volumes of data. We consider a general formulation of a PDE problem

$$
\left\{\begin{array}{l}
\frac{\partial U}{\partial t}(X, t)=\mathscr{M}(U(X, t))+f(X, t) \quad X \in \Omega \quad t \geq 0 \\
\mathscr{C}(U(X, t))=g(t) \quad X \in \partial \Omega \quad t>0 \\
U(X, 0) \quad=U_{0}(X) \quad X \in \Omega .
\end{array}\right.
$$

- $\Omega$ is a bounded domain of $\mathbb{R}^{n} \times \mathbb{R}^{n}$ with the border $\partial \Omega$.

- $X$ is a pair $(x, y)$, where $x \in \mathbb{R}^{n}$ and $y \in \mathbb{R}^{n}$.

- $U(X, t)$ the the unknown function in Hilbert space $\mathscr{H}, f$ an element of $\mathscr{H}$.

- $\mathscr{M}, \mathscr{C}$ are operators from $\mathscr{H}$ on $\mathscr{H}$, where $\mathscr{C}$ define the boundary conditions.

If $\mathscr{M}$ contains partial derivatives of order $k$, the boundary conditions are the number of $k$ ( $g$ with $k$ components). Let $\left(P_{i, j}\right)_{i, j=1 \ldots \infty}$ a twodimensional orthogonal Legendre basis which does not satisfy the boundary conditions.

We search an approximation

$U_{N}(X, t)=\sum_{i=1}^{N} \sum_{j=1}^{N-i} \hat{U}_{i, j} P_{i, j} \quad$ with $\quad \hat{U}_{i, j}=\left\langle U_{N}, P_{i, j}\right\rangle$

in $S_{N}$ (where $S_{N}$ represents the space generated by the $N$ first twodimensional Legendre polynomials) such as

$$
\begin{array}{rlll}
P_{N-k}^{\perp} R_{N} & =0 & & N-k \text { equations } \\
C U_{N} & =0 & k \text { equations }
\end{array}
$$

$-R_{N}$ is the residual function of the spectral decomposition in the basis of Legendre, it is given by

$$
R_{N}=\frac{\partial U_{N}}{\partial t}-\mathscr{M}\left(U_{N}\right)-f
$$

- $P_{N-k}^{\perp} R_{N}$ denotes the orthogonal projection of $\mathscr{H}$ on $S_{N-k}$.

- $S_{N-k}$ space generated by the $N-k$ first two-dimensional Legendre polynomials.

- $U_{N}$ is then determined by $N$ ordinary differential equations.

\subsection{Error Estimation.}

Let $\mathscr{H}$ be a $\mathbb{C}$-Hilbert space, $\mathscr{S}$ a sub-space of $\mathscr{H}$ generated by $(N-k)$ first two-dimensional Legendre polynomials. $\operatorname{dim}(\mathscr{S})=$ $n=(N-k)$, where $k$ represents the number of boundary conditions. Our problem is to find a function $U \in \mathscr{H}$ such that

$$
\left\{\begin{array}{cl}
\frac{\partial U}{\partial t}(X, t)+\mathscr{M}(U(X, t)) & =f \\
\mathscr{C}(U(X, t)) & =g \\
U(0) & =U_{0},
\end{array}\right.
$$

where $f, g$ and $U_{0}$ are given elements of $\mathscr{H}$.

Let $f_{n} \in \mathscr{S}$ be an approximation of $f$, and $U_{n}^{0}$ an approximation of $U_{0}$, then the problem (10) can be rewritten as

$$
\frac{\partial}{\partial t}\left(\mathscr{P} U_{n}\right)+\mathscr{M}\left(U_{n}\right)=f_{n}
$$

where $\mathscr{P}$ represent the Tau projection operator on $\mathscr{S}$. Assume that $\mathscr{S}$ is a normed space and his norm satisfy

$\forall s \in \mathscr{S}, \quad \operatorname{Re}\langle\mathscr{P} s, \mathscr{M} s\rangle \geq \kappa\|s\|^{2}$ and

$\operatorname{Re}\langle f, g\rangle \leq\|f\| \cdot\|g\|$

We define $\tilde{f}_{n}$ by

$$
\tilde{f}_{n}=\frac{\partial}{\partial t}\left(\mathscr{P} \tilde{U}_{n}\right)+\mathscr{M}\left(\tilde{U}_{n}\right),
$$

where $U_{n}$ the solution of the problem (10) and $\tilde{U}_{n}$ any.

Under the assumptions (12) and (13), we can prove the following result

$$
\begin{aligned}
& \left\|\mathscr{M}\left(U_{n}-\tilde{U}_{n}\right)\right\|^{2} \leq\left\|\mathscr{M}\left(U_{n}(0)-\tilde{U}_{n}(0)\right)\right\|^{2}+\frac{1}{\kappa} \\
+ & \int_{0}^{t}\left\|\mathscr{M}^{*}\left(f_{n}-\tilde{f}_{n}(r)\right)\right\|^{2} d r
\end{aligned}
$$

where $\mathscr{M}^{*}$ denote the adjoint of the operator $\mathscr{M}$.

This increase interprets as a result of stability, but it requires some regularity conditions.

Proof. We put

$\frac{\partial}{\partial t}\left(\mathscr{P} U_{n}\right)+\mathscr{M}\left(U_{n}\right)=f_{n} \quad$ and $\quad \frac{\partial}{\partial t}\left(\mathscr{P} \tilde{U}_{n}\right)+\mathscr{M}\left(\tilde{U}_{n}\right)=\tilde{f}_{n}$

then

$$
\mathscr{P}\left(\frac{\partial}{\partial t}\left(U_{n}-\tilde{U}_{n}\right)+\mathscr{M}\left(U_{n}-\tilde{U}_{n}\right)\right)=f_{n}-\tilde{f}_{n} .
$$

Which is multiplied by $\mathscr{M}\left(U_{n}-\tilde{U}_{n}\right)$ for the inner product on the space $\mathscr{S}$

$$
\begin{aligned}
& \left\langle\mathscr{P}\left(\frac{\partial}{\partial t}\left(U_{n}-\tilde{U}_{n}\right)\right), \mathscr{M}\left(\frac{\partial}{\partial t}\left(U_{n}-\tilde{U}_{n}\right)\right)\right\rangle \\
+ & \left\langle\mathscr{M}\left(U_{n}-\tilde{U}_{n}\right), \mathscr{M}\left(\frac{\partial}{\partial t}\left(U_{n}-\tilde{U}_{n}\right)\right)\right\rangle \\
= & \left\langle f_{n}-\tilde{f}_{n}, \mathscr{M}\left(\frac{\partial}{\partial t}\left(U_{n}-\tilde{U}_{n}\right)\right)\right\rangle,
\end{aligned}
$$

whose real part is taken, and since $\frac{\partial}{\partial t}\left(U_{n}-\tilde{U}_{n}\right) \in \mathscr{S}$, it will be

$$
\kappa\left\|U_{n}-\tilde{U}_{n}\right\|^{2} \leq \operatorname{Re}\left\langle\mathscr{P}\left(\frac{\partial}{\partial t}\left(U_{n}-\tilde{U}_{n}\right)\right), \mathscr{M}\left(\frac{\partial}{\partial t}\left(U_{n}-\tilde{U}_{n}\right)\right)\right\rangle .
$$

on the other hand

$$
\begin{aligned}
& \operatorname{Re}\left\langle f_{n}-\tilde{f}_{n}, \mathscr{M}\left(\frac{\partial}{\partial t}\left(U_{n}-\tilde{U}_{n}\right)\right)\right\rangle \\
= & \operatorname{Re}\left\langle\mathscr{M}^{*}\left(f_{n}-\tilde{f}_{n}\right), \frac{\partial}{\partial t}\left(U_{n}-\tilde{U}_{n}\right)\right\rangle \\
\leq & \left(\left\|\mathscr{M}^{*}\left(f_{n}-\tilde{f}_{n}\right)\right\|\right) \cdot\left(\left\|\frac{\partial}{\partial t}\left(U_{n}-\tilde{U}_{n}\right)\right\|\right) \\
\leq & \left(\frac{1}{\sqrt{\kappa}}\left\|\mathscr{M}^{*}\left(f_{n}-\tilde{f}_{n}\right)\right\|\right) \cdot\left(\sqrt{\kappa}\left\|\frac{\partial}{\partial t}\left(U_{n}-\tilde{U}_{n}\right)\right\|\right)
\end{aligned}
$$

Since

$$
\left(\frac{1}{\sqrt{\kappa}}\left\|\mathscr{M}^{*}\left(f_{n}-\tilde{f}_{n}\right)\right\|-\sqrt{\kappa}\left\|\frac{\partial}{\partial t}\left(U_{n}-\tilde{U}_{n}\right)\right\|\right)^{2} \geq 0
$$

then

$$
\begin{aligned}
& \frac{1}{2 \kappa}\left\|\mathscr{M}^{*}\left(f_{n}-\tilde{f}_{n}\right)\right\|^{2}+\frac{\kappa}{2}\left\|\frac{\partial}{\partial t}\left(U_{n}-\tilde{U}_{n}\right)\right\|^{2} \\
\geq & \left(\frac{1}{\sqrt{\kappa}}\left\|\mathscr{M}^{*}\left(f_{n}-\tilde{f}_{n}\right)\right\|\right) \cdot\left(\sqrt{\kappa}\left\|\frac{\partial}{\partial t}\left(U_{n}-\tilde{U}_{n}\right)\right\|\right) \\
\geq & \left(\left\|\mathscr{M}^{*}\left(f_{n}-\tilde{f}_{n}\right)\right\|\right) \cdot\left(\left\|\frac{\partial}{\partial t}\left(U_{n}-\tilde{U}_{n}\right)\right\|\right)
\end{aligned}
$$


then we have

$$
\begin{aligned}
& \operatorname{Re}\left\langle\mathscr{M}^{*}\left(f_{n}-\tilde{f}_{n}\right), \frac{\partial}{\partial t}\left(U_{n}-\tilde{U}_{n}\right)\right\rangle \\
\leq & \frac{1}{2 \kappa}\left\|\mathscr{M}^{*}\left(f_{n}-\tilde{f}_{n}\right)\right\|^{2}+\frac{\kappa}{2}\left\|\frac{\partial}{\partial t}\left(U_{n}-\tilde{U}_{n}\right)\right\|^{2} .
\end{aligned}
$$

The formula (16) becomes

$$
\begin{aligned}
& \kappa\left\|\frac{\partial}{\partial t}\left(U_{n}-\tilde{U}_{n}\right)\right\|^{2}+\frac{1}{2} \frac{\partial}{\partial t}\left\|\mathscr{M}\left(U_{n}-\tilde{U}_{n}\right)\right\|^{2} \\
\leq & \frac{1}{2 \kappa}\left\|\mathscr{M}^{*}\left(f_{n}-\tilde{f}_{n}\right)\right\|^{2}+\frac{\kappa}{2}\left\|\frac{\partial}{\partial t}\left(U_{n}-\tilde{U}_{n}\right)\right\|^{2} \\
\leq & \frac{1}{2 \kappa}\left\|\mathscr{M}^{*}\left(f_{n}-\tilde{f}_{n}\right)\right\|^{2}+\kappa\left\|\frac{\partial}{\partial t}\left(U_{n}-\tilde{U}_{n}\right)\right\|^{2} .
\end{aligned}
$$

hence

$$
\frac{\partial}{\partial t}\left\|\mathscr{M}\left(U_{n}-\tilde{U}_{n}\right)\right\|^{2} \leq \frac{1}{\kappa}\left\|\mathscr{M}^{*}\left(f_{n}-\tilde{f}_{n}\right)\right\|^{2}
$$

by integration of both sides of this inequality we have

$$
\begin{aligned}
\int_{0}^{t} \frac{\partial}{\partial r}\left\|\mathscr{M}\left(U_{n}-\tilde{U}_{n}\right)(r)\right\|^{2} d r & \leq \frac{1}{\kappa} \int_{0}^{t}\left\|\mathscr{M}^{*}\left(f_{n}-\tilde{f}_{n}\right)(r)\right\|^{2} d r \\
\left\|\mathscr{M}\left(U_{n}-\tilde{U}_{n}\right)(t)\right\|^{2} & \leq\left\|\mathscr{M}\left(U_{n}-\tilde{U}_{n}\right)(0)\right\|^{2} \\
& +\frac{1}{\kappa} \int_{0}^{t}\left\|\mathscr{M}^{*}\left(f_{n}-\tilde{f}_{n}\right)(r)\right\|^{2} d r .
\end{aligned}
$$

We assume that $f$ and $U$ are regular functions.

Let $\tilde{U}_{n}$ is an approximation of $U$ such that $\tilde{U}_{n}(0)=U_{n}^{0}$.

Using the increase (14), we can obtain the following error estimation

$$
\begin{aligned}
& \left\|\mathscr{M}\left(U-\tilde{U}_{n}\right)(t)\right\| \\
\leq & \max _{0 \leq r \leq t}\left\|\mathscr{M}\left(U-\tilde{U}_{n}\right)(r)\right\|+\left(\max _{0 \leq r \leq t}\left\|\mathscr{M}^{*}\left(f-f_{n}\right)(r)\right\|\right. \\
+ & \left.\max _{0 \leq r \leq t}\left\|\frac{\partial}{\partial t}\left(U-\tilde{U}_{n}\right)(r)\right\|+\max _{0 \leq r \leq t}\left\|\mathscr{M}^{*} \mathscr{M}\left(U-\tilde{U}_{n}\right)(r)\right\|\right) .
\end{aligned}
$$

Proof. We have

$$
\left\|\mathscr{M}\left(U-U_{n}\right)(t)\right\| \leq\left\|\mathscr{M}\left(U-\tilde{U}_{n}\right)(t)\right\|+\left\|\mathscr{M}\left(U_{n}-\tilde{U}_{n}\right)(t)\right\|,
$$

we can estimate $\left\|\mathscr{M}\left(U_{n}-\tilde{U}_{n}\right)(t)\right\|$ from (14)

$$
\begin{aligned}
\left\|\mathscr{M}^{*}\left(f_{n}-f\right)(t)\right\| \leq & \left\|\mathscr{M}^{*}\left(f-f_{n}\right)(t)\right\|+\left\|\mathscr{M}^{*}\left(f-\tilde{f}_{n}\right)(t)\right\| \\
\leq & \left\|\mathscr{M}^{*}\left(f-f_{n}\right)(t)\right\|+\left\|\mathscr{M}^{*} \frac{\partial}{\partial t}\left(U-\tilde{U_{n}}\right)\right\| \\
& +\left\|\mathscr{M}^{*} \mathscr{M}^{*}\left(U-\tilde{U_{n}}\right)\right\|
\end{aligned}
$$

then

$$
\begin{aligned}
& \left\|\mathscr{M}\left(U-\tilde{U}_{n}\right)(t)\right\| \\
\leq & \max _{0 \leq r \leq t}\left\|\mathscr{M}\left(U-\tilde{U}_{n}\right)(r)\right\|+\left(\max _{0 \leq r \leq t}\left\|\mathscr{M}^{*}\left(f-f_{n}\right)(r)\right\|\right. \\
+ & \left.\max _{0 \leq r \leq t}\left\|\frac{\partial}{\partial t}\left(U-\tilde{U}_{n}\right)(r)\right\|+\max _{0 \leq r \leq t}\left\|\mathscr{M}^{*} \mathscr{M}\left(U-\tilde{U}_{n}\right)(r)\right\|\right) .
\end{aligned}
$$

\section{References}

[1] A. Quarteroni, A. Valli, Numerical Approximations of Partial Differential Equations, (Springer, Heidelberg) 1994

[2] A. Quarteroni, C. Canuto, M. Y. Hussaini, T. A. Zang, Spectral Methods Fundamentals in Single Domains, Springer 2006, ISBN $987-3-540-30725-9$ http : //www.dimat.polito.it/chqz/software.

[3] B. Costa, Spectral Methods for Partial Differential Equations, A Mathematical Journal Vol. 6,No 4,(1-32), december 2004.

[4] B. Costa, L. Dettori, D. Gottlieb and R. Temam,Time Marching Techniques for the Nonlinear Galerkin Method, SIAM Journal of Sci. Comput., Vol. 23(2001), No.1, pp.46-65.

[5] C. Canuto, M. Yousuff Hussaini, Alfio Quarteroni, \& Thomas A. Zang, Spectral Methods in Fluid Dynamics. Springer Series in Computational Physics. Springer, 1987.

[6] J. Boyd, Chebyshev and Fourier Spectral Methods, Second Edition, University of Michigan. 2000. Available online : http://wwwpersonal.umich.edu/ jpboyd/BOOK Spectral2000.html.

[7] M. Druon, Modèlisation du mouvement par polynomes orthogonaux application á l'étude d'ćoulements fluides. $\mathrm{PhD}$ thesis, Universitè de Poitiers, 2009.

[8] S. Orszag, Numerical analysis of spectral methods, Society for Industrial and Applied Mathematics, Philadelphia 1977.

[9] S.A. Orszag, Spectral methods for problems in complex geometries, J. Comput. Phys. 37, 70-92, 1980

[10] Iserles.A A First Course in the Numerical Analysis of Differential Equations 2009

. 